\title{
Performance of graphene ECG electrodes under varying conditions
}

DOI:

10.1109/EMBC.2018.8513376

\section{Document Version}

Accepted author manuscript

Link to publication record in Manchester Research Explorer

\section{Citation for published version (APA):}

Beach, C., Karim, M. N., \& Casson, A. (2018). Performance of graphene ECG electrodes under varying conditions. In IEEE EMBC https://doi.org/10.1109/EMBC.2018.8513376

\section{Published in:}

IEEE EMBC

\section{Citing this paper}

Please note that where the full-text provided on Manchester Research Explorer is the Author Accepted Manuscript or Proof version this may differ from the final Published version. If citing, it is advised that you check and use the publisher's definitive version.

\section{General rights}

Copyright and moral rights for the publications made accessible in the Research Explorer are retained by the authors and/or other copyright owners and it is a condition of accessing publications that users recognise and abide by the legal requirements associated with these rights.

\section{Takedown policy}

If you believe that this document breaches copyright please refer to the University of Manchester's Takedown Procedures [http://man.ac.uk/04Y6Bo] or contact uml.scholarlycommunications@manchester.ac.uk providing relevant details, so we can investigate your claim.

\section{OPEN ACCESS}




\title{
Performance of graphene ECG electrodes under varying conditions
}

\author{
Christopher Beach, Student Member, IEEE, Nazmul Karim, and Alexander J. Casson, Senior Member, IEEE
}

\begin{abstract}
Smart garments for invisible health sensing have been available for a number of years, with heart sensing typically performed using silver loaded conductive threads integrated into the fabric to pick up the electrocardiogram. Recent work has investigated printed graphene textiles as an alternative to this, which are potentially more environmentally friendly, cost-effective, and can be performed after garment manufacturing. This paper presents an exploration of second order factors on the performance of graphene textile electrodes for electrocardiogram measurements. We prepare graphenebased textile electrodes using a simple and highly scalable continuous padding method. We then analyze two metrics: the change in heart rate estimation error, and the changes in signal-to-noise ratio; under two separate conditions: an extended record length, and varying temperatures; to recreate the some of the conditions the material would experience when being worn in real-life. We report that neither the heart rate estimation error or the signal-to-noise ratio are significantly affected after a long record or with varying temperature. These tests indicate that graphene electrodes are suitable for electrocardiogram measurements in a wearable that will be subjected to these conditions.
\end{abstract}

\section{INTRODUCTION}

Wearable monitors are becoming increasingly well known and used to monitor a wide variety of health conditions by both health professionals and consumers. Information on the heart is one of the most important modalities collected by current units, and most current wearable devices measure the heart activity through photoplesmography (PPG). However, this only gives limited information on the heart activity, typically just the heart rate. Wearable Electrocardiogram (ECG) units are starting to appear [1] but placing these at the wrist is challenging [2]. For this reason wearable ECG units often taken the form of smart garments such as T-shirts.

An ECG measures the electrical activity of the heart, and is widely used in clinical settings. An ECG can give more clinical information on the health of the heart, as the signal not only shows each heartbeat, but also the P-wave, T-wave and QRS complex, which may provide more information on the health of the user's heart. Furthermore, from an ECG it is easy to calculate statistics such as the heart rate variability (HRV), which can be used to diagnose atrial fibrillation (AF) [3], as well as in sleep [4], and stress analysis [5].

This work was in part supported by the UK Engineering and Physical Sciences Research Council grant number EP/P02713X/1 and the Doctoral Training Partnership with the University of Manchester.

C. Beach and A. J. Casson are with the School of Electrical and Electronic Engineering, The University of Manchester, UK. Email: christopher.beach@postgrad.manchester.ac.uk, alex.casson@manchester.ac.uk.

N. Karim is with the National Graphene Institute (NGI) and the School of Materials, The University of Manchester, UK. Email: mdnazmul.karimemanchester.ac.uk.
Typical ECG recordings are taken using gel electrodes to reduce the impedance of the connection between the electrode and the body. These electrodes are normally made from $\mathrm{Ag} / \mathrm{AgCl}$, a medical grade material that allows the conversion of the ion signals in the body to an electrical current that can be measured using an ECG amplifier. However, these electrodes are rigid, and the gel dries out over time, limiting their operating lifetime.

To overcome these issues, the usage of conductive textiles in wearable devices for ECG sensing is gaining significant research interest. This is due to their flexibility, providing greater levels of comfort and making devices less obtrusive for the user. This is key when one of the main barriers to the adoption of wearable devices is the obtrusion caused to the wearer [6]. In previous smart textiles, heart sensing has typically been performed using silver loaded conductive threads integrated into the fabric to pick up the electrocardiogram [7]. Recent work has investigated printed graphene on textiles as an alternative to this, which offers benefits of depositing controlled quantities of materials at precise locations, less materials waste and water utilization, and a potentially more environmentally friendly process. This is timely as there have been many advances in the printing of graphene onto flexible substrates such as cotton and nylon, in turn allowing the printing of electronics and sensors directly onto garments. Recent examples of this include printed transistors [8], supercapacitors [9] and pressure sensors [10].

We have previously demonstrated that printed graphene can be utilized for heart rate monitoring [11] by using the printed material as a passive electrode. [11] demonstrates that heart rate can be measured from peripheral sites by resting two fingers onto a flexible graphene based electrode, giving approximately 2 beat-per-minute (bpm) accuracy, using 5 minute recordings under laboratory test conditions. In this paper we extend this previous work to characterize a range of second order factors that would be experienced when these flexible electrodes are used in practice. Here we prepare graphene-based conductive textile electrodes using a simple, scalable and cost-effective padding (coating) technique [12]. While the washability of these materials has been reported previously in terms of change in resistances with number of home laundry washing cycles [12], other factors including temperature variations, stretching, and contorting of the material have not. Additionally, the ability of these smart textiles to provide good quality recordings over extended periods of time is not reported. These factors could potentially cause larger noise on the recordings, making it harder to extract a heart rate reading, therefore increasing the error of heart rate reading taken from a graphene electrode. 
In this work we extend the typical ECG records taken with graphene electrodes to include records taken over much longer record periods (40 minutes compared to the typical 5 minutes), which may cause the user to sweat from a lack of air circulation, altering performance. We also investigate performance over a range of temperatures (rather than just at room temperature). In this paper we analyze the capabilities of the material under these conditions, reporting on two performance metrics: the increase or degradation in signal-tonoise ratio (SNR); and changes in the bpm error in extracting the heart rate from the recorded waveform.

The remainder of this paper is structured as follows. Section II describes the experimental setup used, and Section III reports the performance of the material across a range of temperatures, and results from the ECG heart rate analysis algorithm. Finally conclusions are drawn in Section IV.

\section{METHODS}

\section{A. Preparation of conductive material}

We use a simple and highly scalable continuous process to prepare graphene-coated textile electrodes for ECG measurements. We synthesize reduced graphene oxide (rGO) inks and then coat knitted nylon fabric with rGO using a simple pad-dry process as per our previously reported method [12]. The coated fabrics are dried subsequently at $100{ }^{\circ} \mathrm{C}$ for 10 minutes. Given its conductive properties, it can be used as a passive electrode in ECG recordings. The nylon was cut into two identically sized ( $35 \times 20 \mathrm{~mm})$ electrodes, for fingers from opposite hands. An ECG snap connector (Prym $12 \mathrm{~mm}$ buttons) was fitted onto each electrode as shown in Fig. 1(a), allowing the electrode to be connected to a standard ECG amplifier.

\section{B. Experimental setup}

The same configuration was used for both the extended record test and the temperature test. We used a heat mat from a 3D printer (Lulzbot Mini), with a sheet of glass glued on top to act as a temperature interface to create a thermally stable hot plate. Preliminary experiments found that when using an aluminum sheet glued to the heat mat, the capacitance between the two electrodes was very high, which resulted in saturation of the ECG amplifier, so no signals could be recorded. This occurred even when using a gel mat as a thermal interface on top of the aluminum. Preliminary experiments also identified that when a current was passed through the heat mat to maintain its temperature, a large amount of noise was picked up by the electrodes. This again caused an undesirable effect of affecting the ECG recordings. To ensure the isolation of any outside factors, we opted to heat up the plate to a temperature above the range we wanted to measure, and take a continual reading as the plate cooled down with the heating element disconnected. The large thermal mass of the glass plate ensured that the temperature of the electrodes remained constant over a short period of time, allowing us to calculate bpm error and SNR in set time windows.

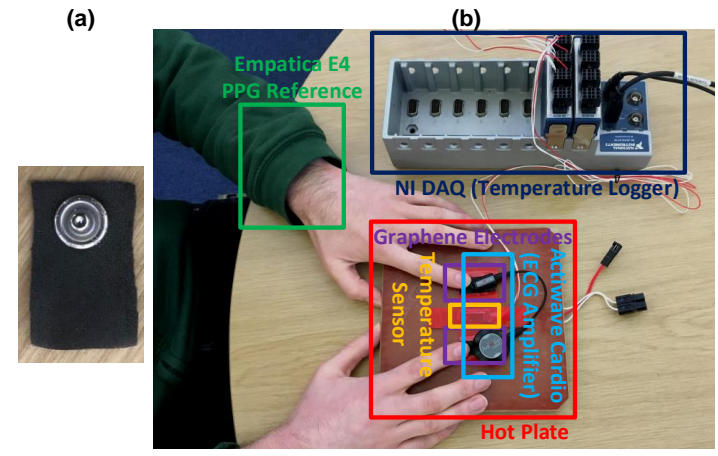

Fig. 1. Experimental setup for taking ECG recordings while varying the temperature of the electrodes.

The electrodes were attached to the hot plate with electrical tape, and the snap connectors connected to an ECG amplifier/recorder (Actiwave Cardio, CamNtech), sampling at $1024 \mathrm{~Hz}$ at $10 \mathrm{bit}$ resolution. Simultaneously, the temperature of the bed was logged in LabVIEW with a DAQ (NI 9219 and NI cDAQ-9178), sampling the temperature every $0.5 \mathrm{~s}$. We logged temperatures between $45-25^{\circ} \mathrm{C}$, in steps of $1{ }^{\circ} \mathrm{C}$ for a period of 40 minutes. In this range the temperature remained constant within $1.3^{\circ} \mathrm{C}$ for 30 seconds, allowing us to take 30 second windows of the record for each degree in temperature decrease. Concurrently, a reference heart rate was taken using a photoplethysmography (PPG) monitor, the Empatica E4, which is a widely used, accurate device. This was worn on the left wrist. PPG was selected as an optical method which will not cause interference with the simultaneous ECG recording, and to allow a comparison bpm measurement to be extracted. The time clocks of all three devices were synchronized at the start of each record. The experimental setup is shown in Fig. 1(b). The institutional review board in Manchester have approved Dr. Casson to perform ECG measurements.

A total of eight, 40 minute records were taken. In four of these the bed was at room temperature, with the temperature remaining constant, within $\pm 2{ }^{\circ} \mathrm{C}$. This provides the results for the extended record test. In the other four records the bed was heated up to $50{ }^{\circ} \mathrm{C}$, and the measurements taken as the bed cooled down, taking measurements continuously when the temperature was between $45-25^{\circ} \mathrm{C}$, as the bed cooled down.

\section{Analysis methods}

For each collected record we analyze both the Signal-toNoise Ratio (SNR) and the bpm error of the ECG against the reference PPG device. To process the ECG record, we apply standard ECG signal processing, the steps of which are described in detail in [2]. In summary, our first step was to remove $50 \mathrm{~Hz}$ mains interference. This was undertaken using a zero phase delay Butterworth filter. To process and find the heart rate of each window, we took the Continuous Wavelet Transform (CWT) with the Mexican hat mother wavelet at scale 0.025 . CWT power coefficients over a specific threshold were taken as candidate R peaks in the ECG trace (which correspond to individual heart-beats). From each of 
these candidate locations in the CWT domain, the actual R peak location in the time domain was found as the maximum signal point within 10 samples of the CWT peak location. Potential spurious peaks which may have arisen from motion artifacts or other external noise were removed. The heart rate in bpm was then calculated. A tracking filter was used to reject any heart rate estimates that were $10 \mathrm{bpm}$ away from the previous estimate, these rejected samples were replaced by using a zero order hold to keep the previous bpm estimate. For some time windows, the mean estimation of the bpm error was very high due to errors from the reference PPG device. These were generally caused by motion artifacts preventing the PPG unit obtaining a good quality signal. In these cases, the time windows were manually adjusted by hand, removing the section with high noise, so that an accurate bpm error could be obtained.

The SNR was calculated throughout the recordings at every $\mathrm{R}$ peak location. The $\mathrm{R}$ peak amplitude was taken as the signal, and the root mean square of the middle third of the ECG trace between consecutive $\mathrm{R}$ peaks taken as the noise. This is illustrated in Fig. 2(b), where the crosses denote the amplitude of the signal (S), and the two circles denote the start and end of the noise $(\mathrm{N})$. The SNR was calculated for each heart beat, and the mean of this was taken for each record.

\section{RESULTS}

\section{A. Signal processing results}

The performance of our graphene electrodes can be seen in Fig. 2. Fig. 2(a) shows an example raw, unfiltered waveform. The signal has a large amount of $50 \mathrm{~Hz}$ noise (the power line frequency in the UK) which prevents clear analysis of the signal. In most cases the R-peaks can still be seen due to their large prominence, but the overall SNR is low and an algorithm to detect heart rate would struggle on this raw data. After running the signal processing steps described in Section II-C, the waveform given in Fig. 2(b) is obtained. Here, the algorithm has automatically identified the R-peak locations, which are indicated by orange crosses, and the SNR is also greatly improved. While in this work we are only interested in extracting the heart rate from the data, the morphological components of the ECG, particularly the T-wave can also be easily be identified, which may prove useful in diagnosis of diseases, which we will discuss in future work. The pop-out figure in Fig. 2(b) shows a close up of one heart-beat, with the P-wave, QRS complex and T-wave clearly visible and highlighted.

Fig. 3 shows how the PPG device is used to provide a reference, allowing us to calculate a heart rate estimation error. In Fig. 3(a) signals from an example record are shown, with both the filtered ECG waveform from the graphene electrodes and the PPG waveform from the reference device. The two waveforms have been synchronized in time with one another, showing how the troughs in the PPG waveform align with the R-peaks, and therefore each heartbeat in the ECG waveform. Fig. 3(b) shows the results of running our heart rate estimation of algorithm on the data in Fig. 3(a).

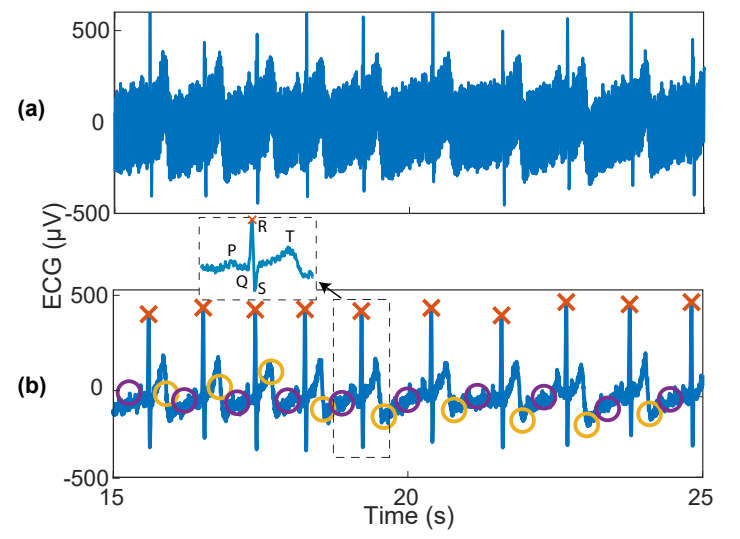

Fig. 2. Example ECG from the graphene electrodes (a) Raw unfiltered waveform (b) Filtered waveform showing R-peak locations (crosses) and noise locations used in SNR calculation (circles). Pop-out figure highlights the P-wave, T-wave, and the QRS complex

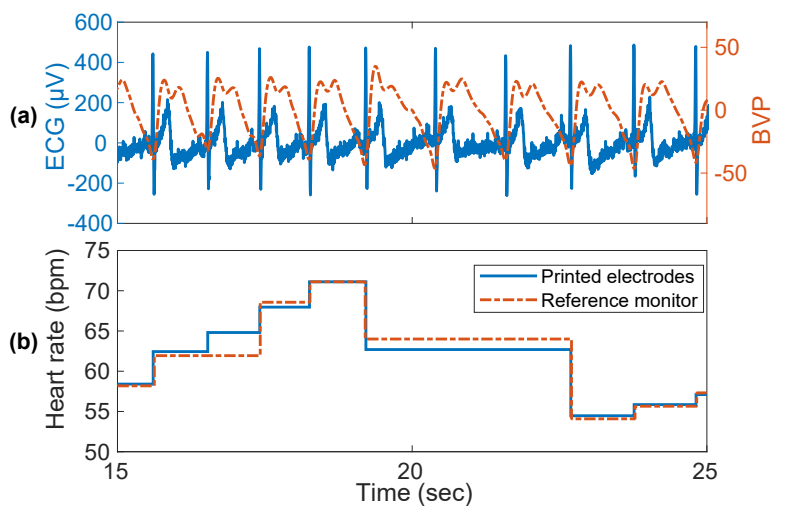

Fig. 3. Sample results showing (a) The filtered ECG waveform from the graphene electrodes, synchronized with the BVP waveform from the reference PPG monitor and (b) The heart rate estimation from both the graphene electrodes and the reference PPG monitor.

Both the calculated heart rate from the graphene electrodes and the heart rate from the reference device are shown. In this example the two follow each other very closely. This was undertaken for the length of the recording and the mean difference between the two bpm methods shown in Fig. 3(b) taken as the mean heart rate estimation error.

\section{B. Extended ECG record results}

Fig. 4 shows the mean heart rate extraction error and SNR for the electrodes during an extended 40 minute record, with the electrodes at the same temperature throughout. Fig. 4(a) shows the variation in the mean heart rate estimation error over the duration of a 40 minute record. Over the test duration the error is typically kept below $3.5 \mathrm{bpm}$, with mean error of $2.0 \mathrm{bpm}$ throughout the record. There are no visible trends in the error throughout the record, with the standard deviation at $1.1 \mathrm{bpm}$. Any spikes in the error shown in Fig. 4(a), are generally due to the loss of a good reference from the PPG device. Fig. 4(b) shows the variation in SNR throughout the record. The SNR is consistently maintained over $25 \mathrm{~dB}$, with an average of $26.4 \mathrm{~dB}$, and a standard deviation of $0.3 \mathrm{~dB}$. Over the duration of the record there is no considerable improvement or degradation of the SNR. These results indicate that when the graphene electrodes are 


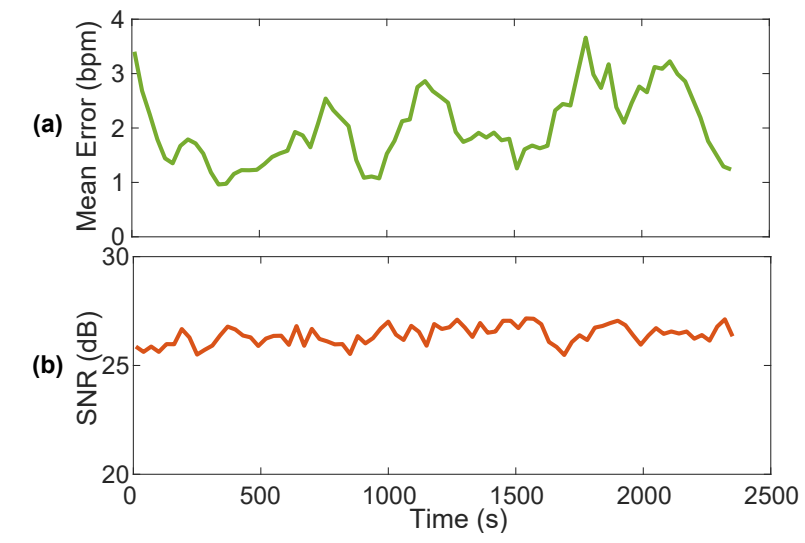

Fig. 4. Mean results from four records for the extended record test, showing variation with time for (a) Mean heart rate estimation error and (b) Signalto-noise Ratio.

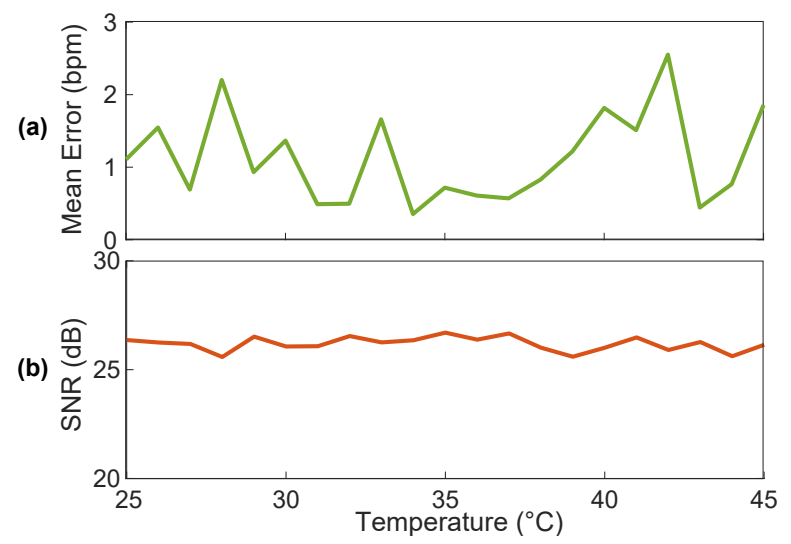

Fig. 5. Mean results from four records showing variation with temperature for (a) Mean heart rate estimation error and (b) Signal-to-noise Ratio.

used for an extended period of time (up to 40 minutes), their performance remains relatively constant, and any sweating from the user from a lack of air circulation at the electrode contact point, does not alter their performance.

\section{Temperature variation results}

Fig. 5 shows the mean heart rate extraction error and SNR for the electrodes while the temperature of the electrodes is varied from $45-25^{\circ} \mathrm{C}$. Fig. 5(a) shows the variation in the mean heart rate estimation error, which is consistently maintained below $3 \mathrm{bpm}$. The mean heart rate estimation error is $1.1 \mathrm{bpm}$, while the standard deviation is $0.6 \mathrm{bpm}$. Across the temperature range there is no trend in the mean heart rate estimation error. Fig. 5(b) shows the variation in SNR as the temperature changed. The SNR is again consistently maintained over $25 \mathrm{~dB}$, with a mean of $26.2 \mathrm{~dB}$, and a standard deviation of $2.4 \mathrm{~dB}$. Across the temperature range there is no considerable improvement or degradation of the SNR. As in the previous test, these results indicate that for the temperature range of $25-45{ }^{\circ} \mathrm{C}$, there is no meaningful change in performance of the electrodes.

\section{CONCLUSIONS}

We have investigated the performance of ECG recordings using graphene electrodes under two test conditions, by monitoring the heart rate estimation error and SNR of each ECG R peak. The first test condition was an extended record length, which was investigated to see if any sweating from the user from a lack of air circulation at the electrode contact point adversely affects the record quality. The second test condition was varying the electrode temperature to again identify if this affects either of the performance metrics. The results indicate that neither of the test conditions substantially affected the performance. This indicates that these smart textiles are still suitable for ECG measurements in a wearable that may be subjected to these second order effects.

\section{REFERENCES}

[1] M. M. Baig, H. Gholamhosseini, and M. J. Connolly, "A comprehensive survey of wearable and wireless ECG monitoring systems for older adults," Med. Biol. Eng. Comput., vol. 51, no. 5, pp. 485-495, 2013.

[2] S. Krachunov, C. Beach, A. J. Casson, et al., "Energy efficient heart rate sensing using a painted electrode ECG wearable," in IEEE GIoTS, Geneva, Jun. 2017.

[3] D. Jabaudon, J. Sztajzel, K. Sievert, et al., "Usefulness of ambulatory 7-day ECG monitoring for the detection of atrial fibrillation and flutter after acute stroke and transient ischemic attack," Stroke, vol. 35, no. 7, pp. 1647-1651, 2004.

[4] M. Bonnet and D. Arand, "Heart rate variability: Sleep stage, time of night, and arousal influences," Clin. Neurophysiol., vol. 102, no. 5, pp. 390-396, 1997.

[5] J. F. Thayer, F. Åhs, M. Fredrikson, et al., "A metaanalysis of heart rate variability and neuroimaging studies: Implications for heart rate variability as a marker of stress and health," Neurosci. Biobehav. Rev., vol. 36, no. 2, pp. 747-756, 2012.

[6] J. S. Abelson, E. Kaufman, M. Symer, et al., "Barriers and benefits to using mobile health technology after operation: A qualitative study," Surgery, vol. 162, no. 3, 2017.

[7] E. P. Scilingo, A. Gemignani, R. Paradiso, et al., "Performance evaluation of sensing fabrics for monitoring physiological and biomechanical variables," IEEE Trans. Inf. Technol. Biomed., vol. 9, no. 3, pp. 345-352, 2005.

[8] T. Carey, S. Cacovich, G. Divitini, et al., "Fully inkjetprinted two-dimensional material field-effect heterojunctions for wearable and textile electronics," Nat. Commun., vol. 8, no. $1202,2017$.

[9] A. M. Abdelkader, N. Karim, C. Vallés, et al., "Ultraflexible and robust graphene supercapacitors printed on textiles for wearable electronics applications," 2D Materials, vol. 4, no. 3, 2017.

[10] H. Tian, Y. Shu, X.-F. Wang, et al., "A graphene-based resistive pressure sensor with record-high sensitivity in a wide pressure range," Sci. Rep., vol. 5, no. 8603, 2015.

[11] N. Karim, S. Afroj, A. Malandraki, et al., "All inkjetprinted graphene-based conductive patterns for wearable etextile applications," J. Mater. Chem. C, vol. 5, no. 44, pp. $11640-11648,2017$.

[12] N. Karim, S. Afroj, S. Tan, et al., "Scalable production of graphene-based wearable e-textiles," ACS Nano, vol. 11, no. 12, pp. 12 266-12275, 2017. 